\title{
Autophagy inhibition enhances RAD00 I-induced cytotoxicity in human bladder cancer cells
}

\author{
This article was published in the following Dove Press journal: \\ Drug Design, Development and Therapy \\ 18 April 2016 \\ Number of times this article has been viewed
}

\author{
Ji-Fan Lin' \\ Yi-Chia Lin ${ }^{2,3}$ \\ Shan-Che Yang' \\ Te-Fu Tsai ${ }^{2,3}$ \\ Hung-En Chen ${ }^{2}$ \\ Kuang-Yu Chou ${ }^{2,3}$ \\ Thomas I-Sheng Hwang ${ }^{2-4}$ \\ 'Central Laboratory, Shin-Kong \\ Wu Ho-Su Memorial Hospital, \\ Taipei, Taiwan; ${ }^{2}$ Division of Urology, \\ Department of Surgery, Shin-Kong \\ Wu Ho-Su Memorial Hospital, \\ Taipei, Taiwan; ${ }^{3}$ Division of Urology, \\ School of Medicine, Fu-Jen Catholic \\ University, New Taipei City, Taiwan; \\ ${ }^{4}$ Department of Urology, Taipei \\ Medical University, Taipei, Taiwan
}

Correspondence: Thomas I-Sheng Hwang Division of Urology, Department of Surgery, Shin Kong Wu Ho-Su Memorial Hospital, 95 Wenchang Road, Shilin

District, Taipei II I0I, Taiwan

Tel +88622833221I ext 2065

Fax +886228389404

Email M001009@ms.skh.org.tw
Background: Mammalian target of rapamycin (mTOR), involved in PI3K/AKT/mTOR pathway, is known to play a central role in regulating the growth of cancer cells. The PI3K/AKT/mTOR pathway enhances tumor survival and proliferation through suppressing autophagy, which sustains energy homeostasis by collecting and recycling cellular components under stress conditions. Conversely, inhibitors of the mTOR pathway such as RAD001 induce autophagy, leading to promotion of tumor survival and limited antitumor efficacy. We thus hypothesized that the use of autophagy inhibitor in combination with mTOR inhibition improves the cytotoxicity of mTOR inhibitors in bladder cancer.

Materials and methods: The cytotoxicity of RT4, 5637, HT1376, and T24 human bladder cancer cells treated with RAD001 alone or combined with autophagy inhibitors (3-methyladenine (3-MA), bafilomycin A1 (Baf A1), chloroquine, or hydroxychloroquine) was assessed using the WST-8 cell viability kit. The autophagy status in cells was analyzed by the detection of microtubule-associated light chain 3 form II (LC3-II), using immunofluorescent staining and Western blot. Acidic vesicular organelle (AVO) formation in treated cells was determined by acridine orange vital staining. Inhibition of mTOR pathway by RAD001 was monitored by using a homemade quantitative polymerase chain reaction gene array, while phospho-mTOR was detected using Western blot. Induced apoptosis was determined by measurement of caspase 3/7 activity and DNA fragmentation in cells after treatment.

Results: Advanced bladder cancer cells (5637, HT1376, and T24) were more resistant to RAD001 than RT4. Autophagy flux detected by the expression of LC3-II showed RAD001induced autophagy. AVO formation was detected in cells treated with RAD001 and was inhibited by the addition of 3-MA or Baf A1. Cotreatment of RAD001 with autophagy inhibitors further reduced cell viability and induced apoptosis in bladder cancer cells.

Conclusion: Our results indicate that simultaneous inhibition of the mTOR and autophagy pathway significantly enhances apoptosis, and it is suggested to be a new therapeutic paradigm for the treatment of bladder cancer.

Keywords: autophagy, apoptosis, bladder cancer, chloroquine, RAD001

\section{Introduction}

Bladder cancer is one of the most common noncutaneous malignancies with high mortality in general population, particularly in men. ${ }^{1}$ Approximately, one-third of patients diagnosed with high-risk non-muscle-invasive bladder cancer are expected to have progression to advanced disease within 5 years. ${ }^{2}$ Radical cystectomy is a common clinical modality for advanced bladder cancer, but it fails to increase survival rates due to recurrences and metastases. ${ }^{3}$ To improve survival rates, a novel chemotherapeutic strategy against bladder cancer is in an urgent need, which prevents and/or delays progression of bladder cancer. 
Activation of mammalian target of rapamycin (mTOR) signaling has been demonstrated in aggressive cancers such as gastric and cervical cancer. ${ }^{4-6}$ The effect of mTOR signaling has also been evaluated in bladder cancer. ${ }^{7}$ The expression of phosphor-S6 (a marker of mTOR activity) was found in $55 \%$ of muscle-invasive bladder cancers with evident lymph node metastases. mTOR activity was demonstrated to be associated with increased pathological stage and reduced patient survival. Inhibition of mTOR using rapamycin-reduced tumor volume in a T24-xenograft model was also observed. ${ }^{7}$ Therefore, targeting mTOR is a reasonable strategy to manage bladder cancer. RAD001 is an mTOR inhibitor that shares similar pharmacological activity with rapamycin as a chemotherapeutic agent. ${ }^{8}$ RAD001 has been demonstrated to exert antiproliferative activities against human breast cancer cell lines, gastric cancer, lymphoma cells, and prostate cancer cells. ${ }^{9-12}$ Its antitumor effects on different solid cancers such as renal and endometrial cancers are under clinical evaluation. ${ }^{13,14}$ It has a relatively short half-life and favorable bioavailability. RAD001 specifically binds with FKBP-12 after entering cells, forming a complex that binds to mTOR. ${ }^{15}$ RAD001 thus inhibits growth factorinduced transduction signaling that is involved in cellular division, cell cycle, and apoptosis. ${ }^{16}$

The aim of this study was to investigate the mechanism underlying the anticancer effects of RAD001 in different grades of bladder cancer cells. We found that RAD001 induced autophagy in bladder cancer cells and that sensitivity to RAD001 was associated with the grade of cancer cells. Furthermore, the inhibition of autophagy could lead to the enhancement of RAD001-mediated cytotoxicity.

\section{Materials and methods Chemicals}

RAD001 was purchased from Sigma-Aldrich (St Louis, MO, USA). A $10 \mathrm{mM}$ stock solution for cell culture was prepared in dimethyl sulfoxide (DMSO), and aliquots were stored at $-20^{\circ} \mathrm{C}$. All other chemicals, unless otherwise mentioned, were purchased from Sigma-Aldrich.

\section{Cell culture}

Human bladder cancer cell lines RT4 (American Type Culture Collection, ATCC, Manassas, VA, USA; \#HTB-2), 5637 (ATCC\#HTB-9), HT1376 (ATCC\#CRL-1472), and T24 (ATCC\#HTB-4) were obtained from Bioresource Collection and Research Center (Hsinchu, Taiwan) and maintained at $37^{\circ} \mathrm{C}$ under $5 \% \mathrm{CO}_{2}$. RT4 and HT1376 cells were cultured in McCoy's 5A and minimum essential medium, respectively. 5637 and T24 cells were cultured in Roswell Park Memorial
Institute-1640 medium. Culture media were supplemented with 10\% fetal bovine serum (Thermo Fisher Scientific, Waltham, MA, USA), 2 mM GlutaMAX-1 (Thermo Fisher Scientific), 100 units/mL penicillin, and $100 \mu \mathrm{g} / \mathrm{mL}$ streptomycin (Thermo Fisher Scientific). Cells were treated with the indicated concentration of RAD001 with or without the treatment with autophagy inhibitors, including bafilomycin A1 (BafA1), 3-methyladenine (3-MA), and chloroquine (CQ), while control cells received an equal volume of DMSO. The final concentration of DMSO was less than $0.1 \%$. Ethical permission for the use of cell lines was not necessary for this study, as only patients' samples or animal research requires such approval from the institutional review board or relevant ethical committee.

\section{Cell viability assays}

The effect of RAD001 on the growth of human bladder cancer cells was examined using the WST-8 reagent (SigmaAldrich) as previously described ${ }^{17}$ In some experiments, cell viability was detected in RAD001-treated cells cotreated with autophagy inhibitors, including 3-MA, Baf A1, or CQ.

\section{Detection of mTOR-related genes by quantitative polymerase chain reaction}

Genes involved in mTOR signaling, including mTOR complexes (MLST8 and RPTOR (mTOR complex 1 [mTORC1]) and RICTOR (mTOR complex 2 [mTORC2])), mTORC1positive regulation (EIF4B, RPS6, and RPS6KB1), and mTORC2-positive regulation (AKT1, GSK3B, and RPS6KB1) were detected using quantitative polymerase chain reaction (qPCR). Total RNA was extracted, using TRIZol reagent (Thermo Fisher Scientific), from T24 cells treated with $1 \mu \mathrm{M}$ RAD001 for 24 hours. RNA yields were measured using Nanodrop 2000 (Thermo Fisher Scientific). Two micrograms of total RNA was subjected to reverse transcription using SuperScript III First-Strand Synthesis System (Thermo Fisher Scientific). qPCR was performed as previously described. ${ }^{18}$ GAPDH was employed as internal control. Relative expression was calculated by the comparative $\mathrm{Ct}$ and expressed as fold of control. Sequences of primer pairs used in this study are listed in Table S1.

\section{Detection of mTORCI downstream effectors by Western blot}

Cells were harvested and lysed using radioimmunoprecipitation assay buffer, followed by measurement of protein concentration using a BCA protein assay (Pierce, Rockford, IL, USA). Protein samples were subjected to a $14 \%$ sodium dodecyl sulfate polyacrylamide gel electrophoresis and subsequently transferred to polyvinylidene fluoride (PVDF) membranes 
(Millipore, Billerica, MA, USA). Antibodies against RPS6 and phospho-RPS6 (p-RPS6) were purchased from Cell Signaling Technology (Danvers, MA, USA); antibody against $\beta$-actin served as internal control and was obtained from Sigma-Aldrich. Immunoblotting procedures were performed using a chemiluminescence procedure (Millipore) per the manufacturer's instructions. The intensity of immunoreactive bands were determined using GeneTools software (Syngene, Frederick, MD, USA) after scanning the chemiluminescence signals on the membrane by a Multigel-2 multifunction gel image system (TOP BIO CO., Taipei, Taiwan). Results are presented as mean \pm standard deviation of three independent experiments.

\section{Autophagy assays}

Induction of autophagy was monitored in cells treated with indicated concentration of RAD001 with or without 2 hours pretreatment with $200 \mathrm{nM}$ Baf A1 by the formation of acidic vesicular organelles using acridine orange staining as described; ${ }^{19}$ and the detection of microtubule-associated protein 1B-light chain 3 (LC3) processing using Western blot as described. ${ }^{17}$

\section{Caspase- $3 / 7$ activity}

Caspase-3 activity was assayed using (H-Asp-Glu-Val-Asp) Rhodamine 110 ((Z-DEVD) 2 -R110, Bachem, Torrance, CA, USA) substrate as previously described. ${ }^{17,18}$ In brief, cells were seeded in 96-well plates and incubated overnight. The cells were then treated with DMSO (control) or $1 \mu \mathrm{M}$ RAD001 with or without the 2 hours pretreatment of inhibitors (1 mM 3-MA or $200 \mathrm{nM}$ Baf A1) for 48 hours. The cells were subsequently lysed with caspase-3 assay buffer containing (Z-DEVD) R110 substrates and incubated at $37^{\circ} \mathrm{C}$ for 1 hour. The fluorescent intensity of proteolytically released fluorophore R110 was then measured using a plate reader (Victor X2; PerkinElmer, Waltham, MA, USA) with an excitation wavelength of $485 \mathrm{~nm}$ and emission wavelength of $535 \mathrm{~nm}$.

\section{Statistical analysis}

All data are expressed as the mean \pm standard deviation. The statistical significance of the differences in variables between groups was calculated using Student's $t$-test, and $P<0.05$ was considered statistically significant. Each experiment was performed at least in triplicate.

\section{Results}

\section{RAD00I inhibits the growth of bladder cancer cells}

The effect of RAD001 on the survival of bladder cancer cell lines was determined using WST-8 assay. Treatment of bladder cancer cell lines with RAD001 resulted in increased cell death in a dose-dependent manner (Figure 1). Our data revealed that RAD001 exerted inhibitory effects on RT4 cells (Grade I with wild-type p53) at a concentration as low as $50 \mathrm{nM}$ at 24 hours, whereas the other bladder cell lines had no response to the treatment (Figure 1). Advanced bladder cancer cells, HT1376, 5637, and T24, were found responding to the RAD001 treatment at concentrations of over $20 \mu \mathrm{M}$. Prolonged exposure to low concentrations of RAD001 had a greater effect on the survival of 5637 and HT1376 cells (Grade II transitional cell carcinoma [TCC] with mutant p53) compared with that of T24 cells (Figure 1B). Given the findings, the concentration of RAD001 at 1 and $5 \mu \mathrm{M}$ was used for following experiments.

\section{RAD00I induces autophagy in bladder cancer cells}

We next examined the effects of RAD001 on mTOR signaling in advanced bladder cancer cells. q-PCR was performed to analyze the levels of mTOR-related genes after a single 24-hour exposure of T24 cells to 1 or $5 \mu \mathrm{M}$ of RAD001. As shown in Figure 2, RAD001 treatment resulted in a downregulated expression of mTOR-related genes (Figure 2A), including RPS6 and RPS6KB1, which are positively regulated by mTORC1 (mTOR-positive regulation; Figure 2B). It is known that RAD001 has an effect on mTORC1 but not on mTORC2 protein complex signaling. ${ }^{20}$ Interestingly, the results revealed that RAD001 induced a downregulation of mTORC2-related gene expression, such as those of AKT1 and RPS6KB1 (mTORC2-positive regulation; Figure 2C). However, AKT1 expression was increased in cells treated with $5 \mu \mathrm{M}$ RAD001 compared to those cells treated with $1 \mu \mathrm{M}$ RAD001 at 24 hours posttreatment (Figure 2C). We further detected the expression of the downstream effector of mTORC1, RPS6; the results showed that RAD001 potently inhibited RPS6 activation throughout the duration of treatment (Figure 2D).

To investigate the role of autophagy in RAD001-induced cytotoxicity, fluorescent imaging was employed to morphologically assess the formation of autophagosome in bladder cancer cells. As shown in Figure 3A, treatment with $1 \mu \mathrm{M}$ of RAD001 for 24 hours resulted in an accumulation of lysosomal vacuoles exhibiting autolysosomal characteristics, whereas the control cells exhibited few vacuoles in number. Western blotting showed that RAD001 treatment increased the breakdown of p62 (also known as SQSTM1, a polyubiquitin-binding protein that is degraded by autophagy) ${ }^{21}$ and LC3-I, with a corresponding increase in LC3-II level in a dose-dependent manner in T24 cells (Figure 3B). These results indicated that RAD001 induces autophagy in human bladder cancer cells. Administration of autophagy inhibitor, Baf A1, significantly 

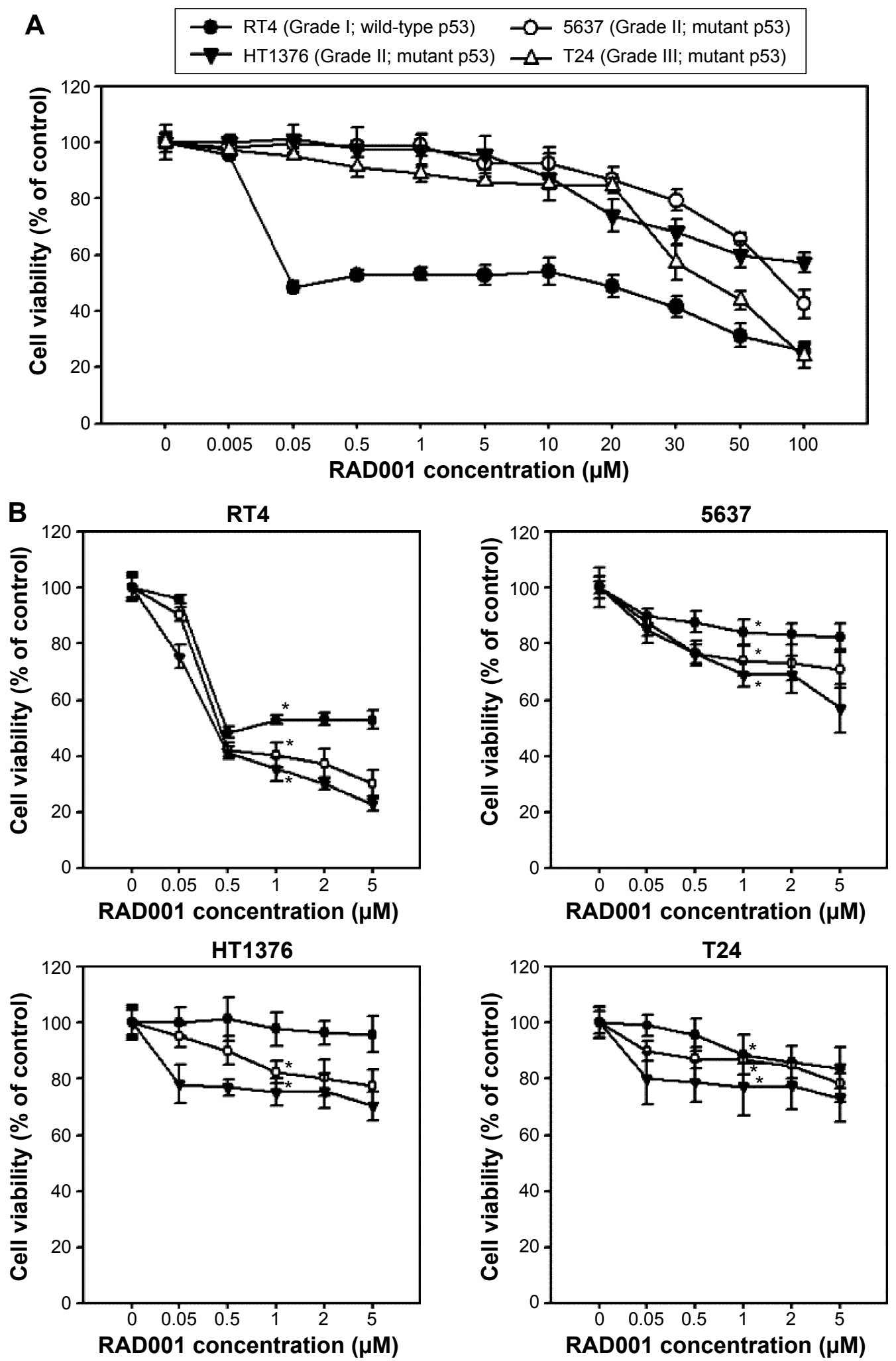

$\rightarrow-24$ hours $\rightarrow-0$ hours $\rightarrow-72$ hours

Figure I RAD00I decreased cell viability in human bladder cancer cells.

Notes: (A) Cell viability of bladder cancer cells treated with various concentration of RAD00I for 24 hours. Cells were seed in 96 -well plates for 24 hours before the addition of indicated concentration of RAD00I. The cell viability was then detected at 24 hours of treatment using WST-8 cell viability kit. Four bladder cancer cell lines were used: RT4 (Grade I TCC; wild-type p53), 5637 (Grade II TCC; mutant p53), HTI 376 (Grade III TCC; mutant p53), and T24 (Grade III TCC; mutant p53). (B) Cell viability of RT4, 5637, HTI376, and T24 cells upon RAD00I treatment for 24,48 , and 72 hours. Cell viabilities of these cells treated with $0-5 \mu M$ RAD00I as described in (A). The values are shown as the mean \pm SD of three independent experiments; $* P<0.05$.

Abbreviations: SD, standard deviation; TCC, transitional cell carcinoma. 
A
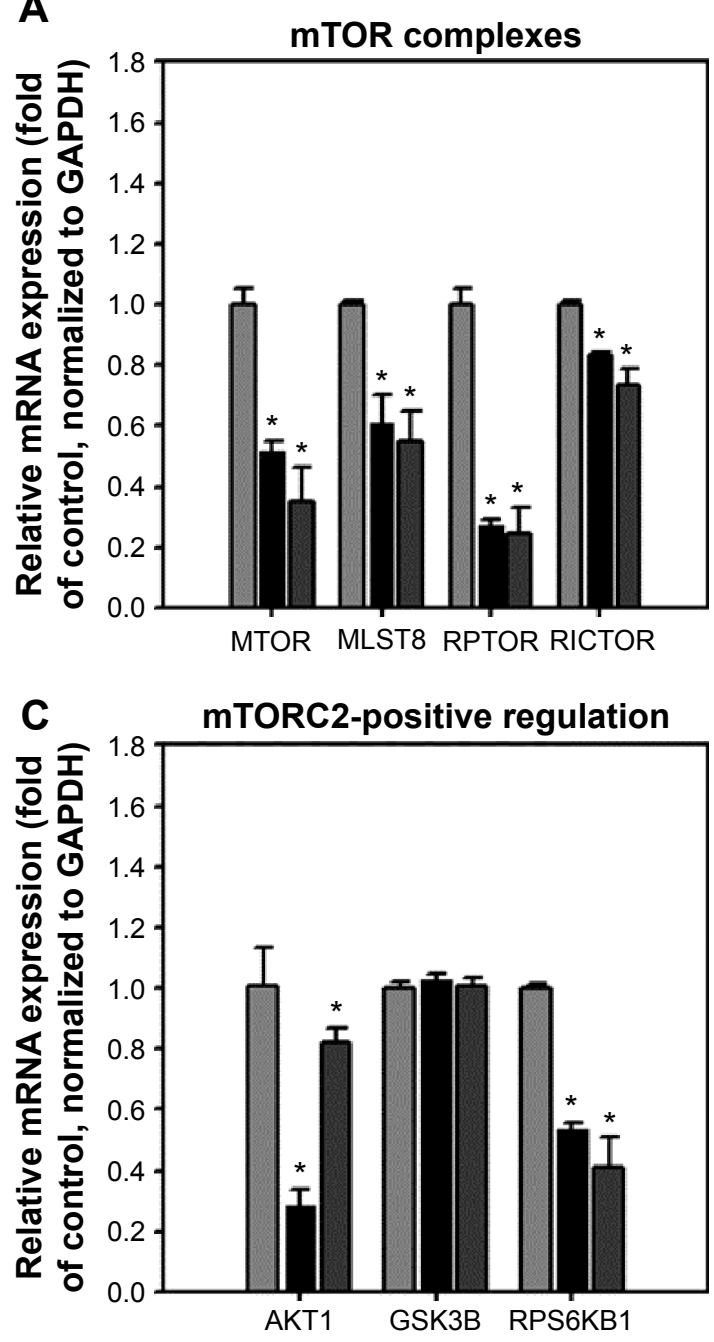

B

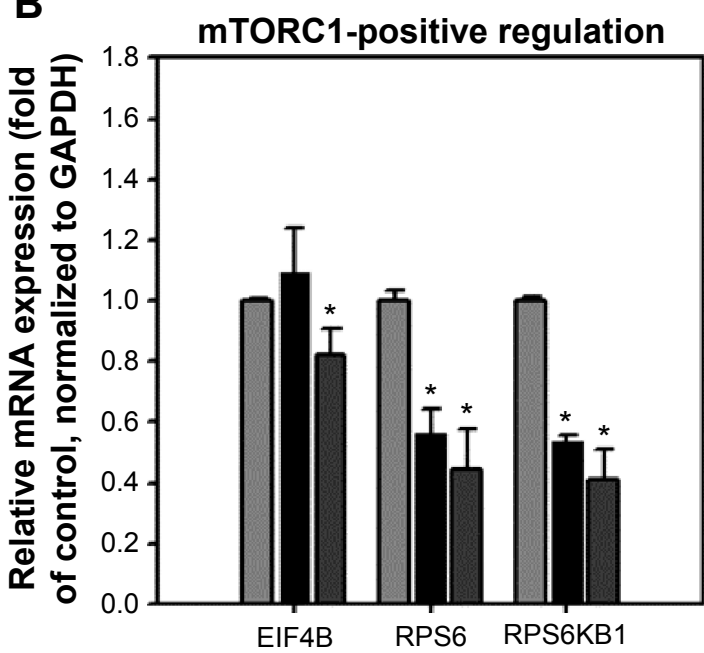

D

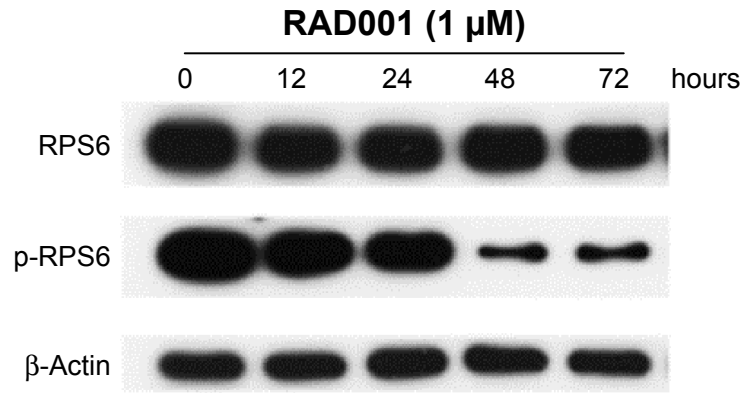

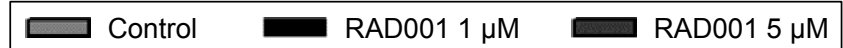

Figure 2 Inhibition of mTOR-related genes and mTORCI downstream effectors by RAD00I.

Notes: qPCR detection of mTOR-related genes, including (A) mTOR complexes, (B) mTORCl-positive regulation, and (C) mTORC2-positive regulation in T24 cells treated with I or $5 \mu$ M RAD00I. Cells were seeded 24 hours before RAD00I treatment. Total RNA samples were extracted at 24 hours posttreatment, reverse transcribed, and subjected to the detection of genes involved in mTOR signaling. The values are shown as the mean \pm SD of three independent experiments; *P $<0.05$. (D) Protein samples were isolated at indicated time points then subjected to the detection of p-4EBPI and p-RPS6 by Western blot.

Abbreviations: mTOR, mammalian target of rapamycin; SD, standard deviation; p-4EBPI, phospho-4EBPI; p-RPS6, phospho-RPS6; mTORCI, mTOR complex I; mTORC2, mTOR complex 2; qPCR, quantitative polymerase chain reaction; mRNA, messenger RNA.

increased the accumulation of p62 and LC3-II protein, suggesting that RAD001 induces a continuous autophagic flux in T24 cells (Figure 3C). The data of Western blotting confirmed the inhibition of autophagy in the presence of Baf A1 in bladder cancer cells by blocking autophagolysosome-mediated degradation of p62 and LC3-II protein.

\section{Inhibition of autophagy enhances RAD00I-induced cytotoxicity in bladder cancer cells}

We next examined whether the inhibition of autophagy sensitizes bladder cancer cells to RAD001-induced cell death using WST-8 assay. As presented in the "RAD001 inhibits the growth of bladder cancer cells" section, sensitivity of bladder cancer cells to RAD001 is suggested to be related to the grade of the tumor. Therefore, three bladder cancer cell lines were exposed to RAD001 in the absence or presence of autophagy inhibitors (including 3MA, Baf A1, or CQ). The low-grade bladder cancer cell line, RT4, exhibited a significantly decreased viability in the presence of a combination of RAD001 and autophagy inhibitor compared with that of RAD001 only (Figure 4A). The data revealed that the viability of bladder cancer cells was significantly decreased in the presence of RAD001 and autophagy inhibitor compared with that exposed only to RAD001. In high-grade bladder cancer cell lines, a significant difference in cell viability between the 
absence and presence of late-stage autophagy inhibitor was observed at high concentrations (Figure 4B and C).

\section{Autophagy inhibition triggers apoptosis in bladder cancer cells}

We demonstrated that cytotoxicity of RAD001 in bladder cancer cells was enhanced in response to cotreatment with autophagy inhibitors. It is of interest to elucidate the mechanism by which the combination of mTOR inhibitor and autophagy inhibitor cause cell death in bladder cancer cells. Treatment of RT4 with $1 \mu \mathrm{M}$ of RAD001 resulted in a significant increase in caspase $3 / 7$ activity, while the induction of apoptosis was absent in 5637 and T24 cell lines. The combination of RAD001 and 3MA or Baf A1 led to a substantial elevation of caspase 3/7 activity in 5637 and T24 cell lines compared with that in the absence of autophagy inhibitors (Figure 5). These data suggested that inhibition of autophagy significantly enhanced RAD001-induced apoptosis in high-grade bladder cancer cells.

\section{Discussion}

In this study, we reported that RAD001 treatment induced autophagy in bladder cancer cells. We found that a decreased sensitivity to RAD001 was associated with high-grade bladder cancer. Furthermore, coordinate inhibition of mTOR and autophagy led to enhanced cell death in high-grade bladder cancer cells.

The mTOR protein is known for its role in regulation of cell growth and metabolic processes. Deregulated mTOR signaling pathway has been shown to contribute to the development of many disorders including cancer. ${ }^{22}$ mTOR protein forms two distinct complexes, mTORC1 and mTORC2. The mTORC1 is the nutrient-sensitive complex consisting of the mTOR interacting protein raptor. It is involved in cell growth and cell size by regulating protein synthesis and autophagy. ${ }^{23}$ Upon activation, mTORC1 phosphorylates the translational regulator eukaryotic translation initiation factor 4E (eIF4E) binding protein 1 (4E-BP1) and S6 kinase (S6K). mTORC1 inhibition has been shown to impair protein synthesis and alter gene transcription. ${ }^{24}$ In this study, we demonstrated that the mTORC1 inhibitor RAD001 exerts anticancer activity in bladder cancer cells. It is suggested that mTOR inhibition by RAD001 is effective in disturbing the growth of bladder cancer cells. However, the sensitivity to RAD001 varied among the bladder cancer cell lines tested, with RT4 cells being the most responsive and T24 cells being the least responsive. It has been reported that cellular response to mTORC1 inhibition is associated with the p53 status in many types of tumor. ${ }^{25-27}$ Recent studies have suggested that rapamycin-mediated tumor suppression is dependent on $\mathrm{p} 53$, which reduces cellular proliferation after mTORC1 inhibition. ${ }^{25,28}$ Our findings that RT4 cells with wild-type p53 were more sensitive to RAD001 than the other ones with mutated $p 53$ support the results of previous studies, implying a heterogeneous response to RAD001-mediated mTOR inhibition. Although inhibition of mTORC1 was expected to diminish cancer cell survival, the extent of cytotoxicity can be reduced by additional changes that occur. For example,
A
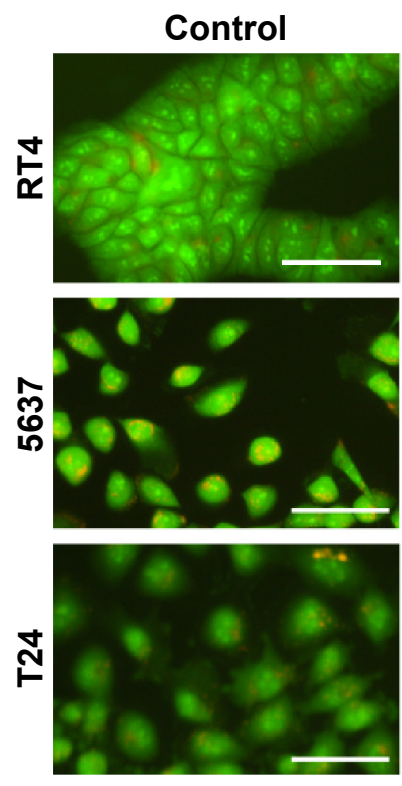

RAD001 $1 \mu M$
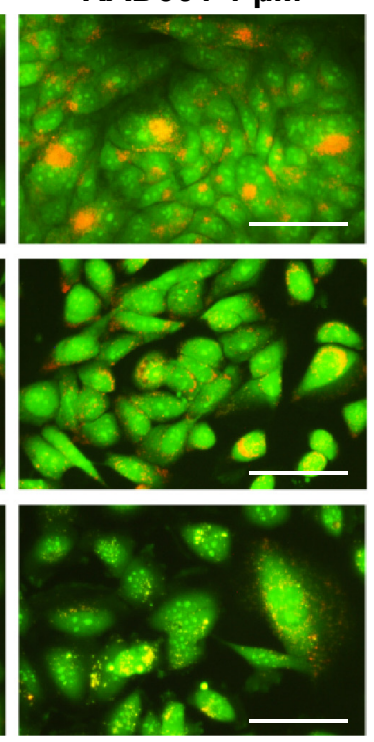

Baf A1 $200 \mathrm{nM}$ RAD001 $1 \mu \mathrm{M}$
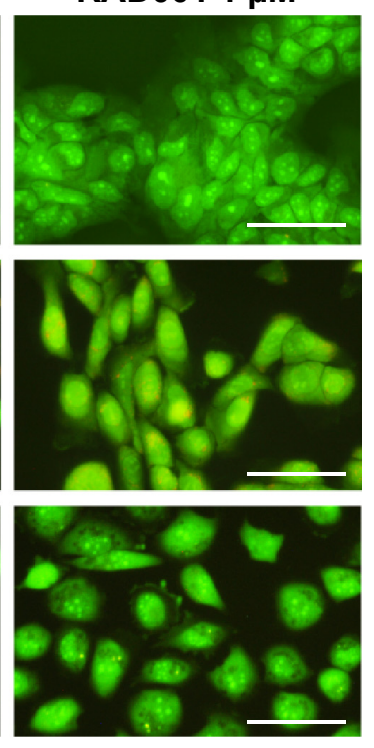

B

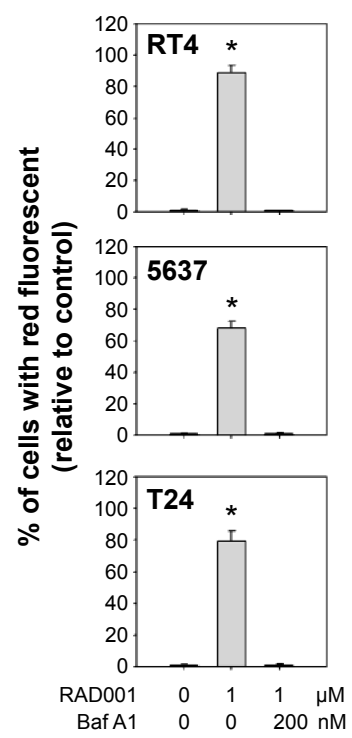

Figure 3 (Continued) 
C
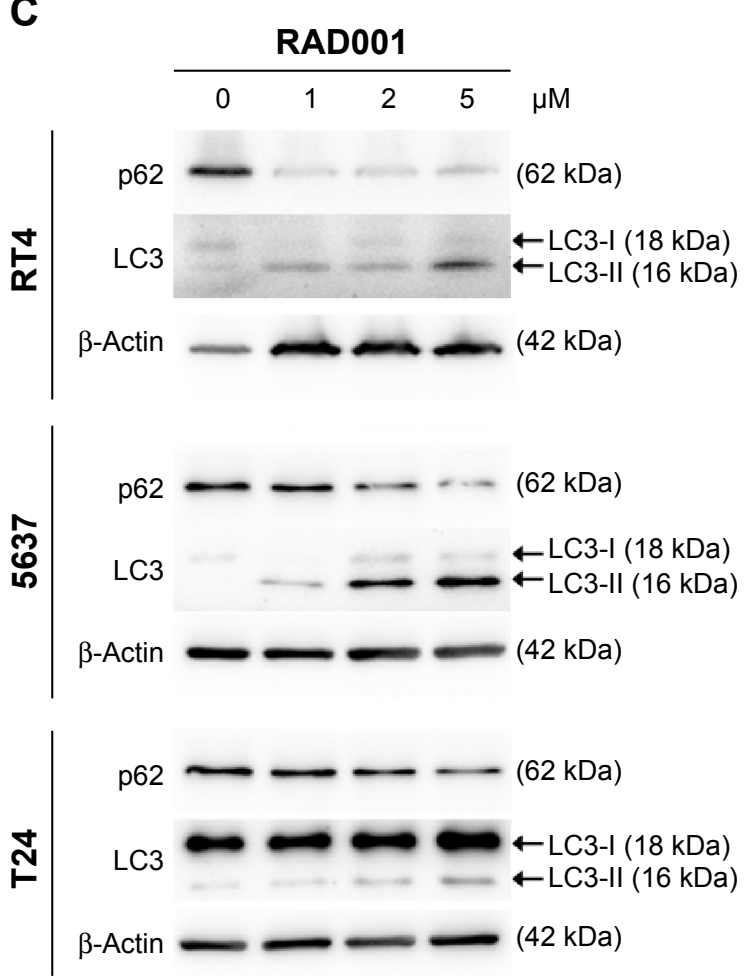
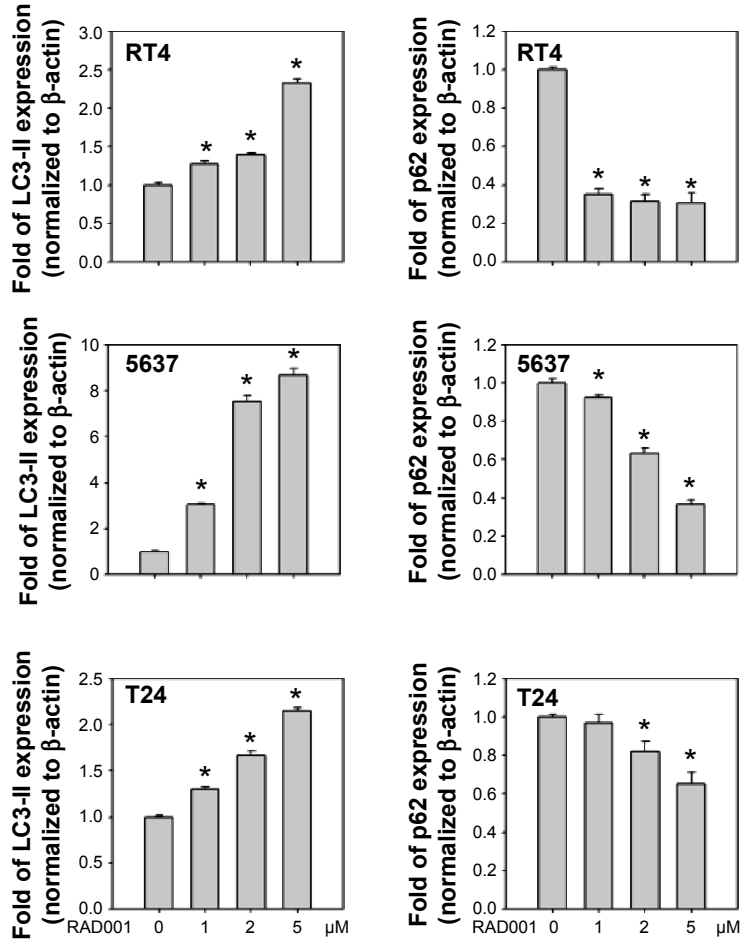

\section{D}
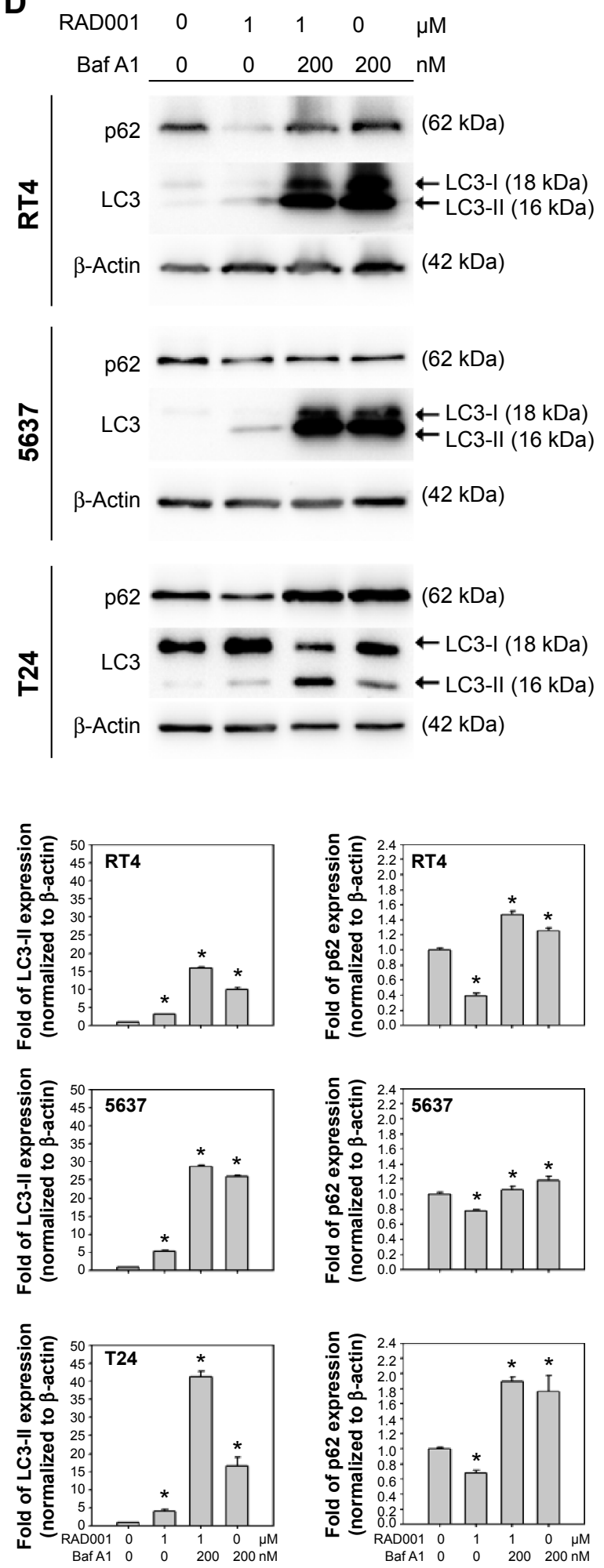

Figure 3 RAD00I induces autophagy in human bladder cancer cells.

Notes: (A) Detection of AVO formation by AO vital staining in RT4, 5637, and T24 cells treated with I $\mu$ M RAD00I with or without 2 hours pretreatment of 200 nM Baf AI. Representative photos from three independent experiments with similar results are shown. Scale bar: $50 \mu \mathrm{m}$. (B) Quantitative detection of AVO formation was determined using flow cytometry. Treated cells were collected immediately after AO staining and the percentage of red-fluorescent-positive cells compared to control was measured using flow cytometry. Data are from three independent experiments with similar results and are presented as mean \pm SD; $* P<0.05$. (C) Detection of LC3-II processing and (D) autophagic flux was detected by monitoring the expression level of p62 and LC3-II in cells treated with 0-5 $\mu$ M RAD00I for 24 hours and cells treated with I $\mu$ M RAD00I with or without the cotreatment of $200 \mathrm{nM}$ Baf AI, respectively. The relative band intensities of p62 and LC3-II were quantitated by denstometric scanning and the relative expression levels are presented as the fold of control cells (lower panels in $\mathbf{C}$ and $\mathbf{D}$ ). The statistical calculation from blots of three independent experiments is shown. The results are presented as the mean $\pm S D ; * P<0.05$.

Abbreviations: AVO, acidic vesicular organelle; AO, acridine orange; SD, standard deviation; Baf AI, bafilomycin AI. 
A

RT4

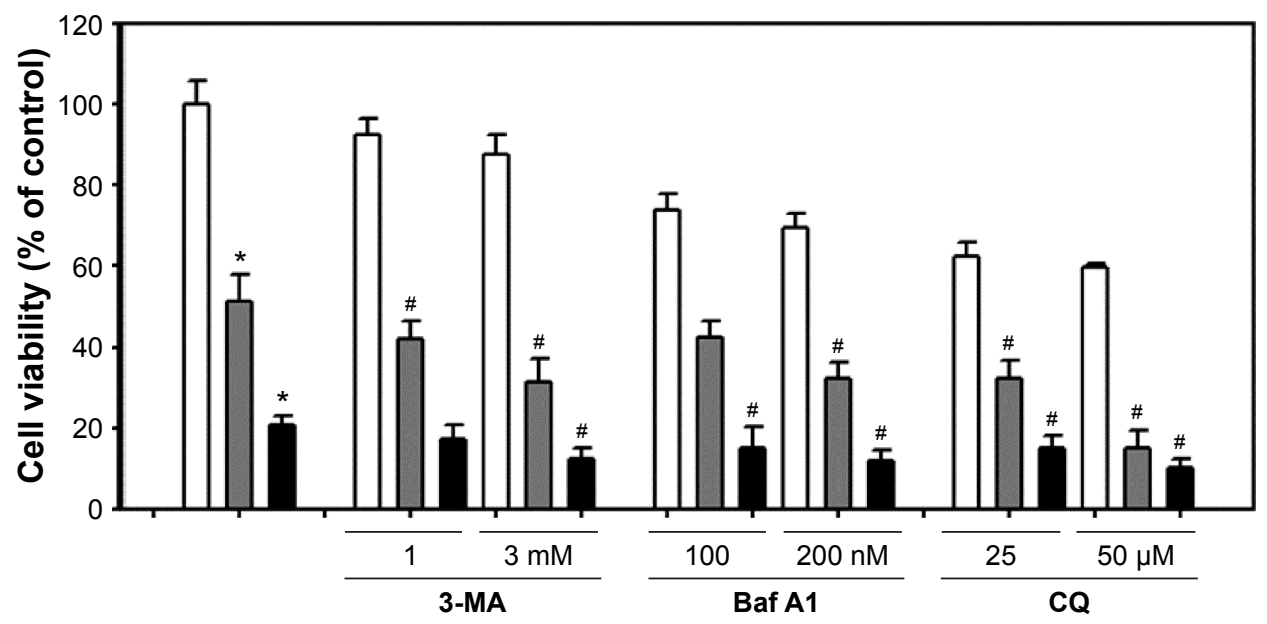

B

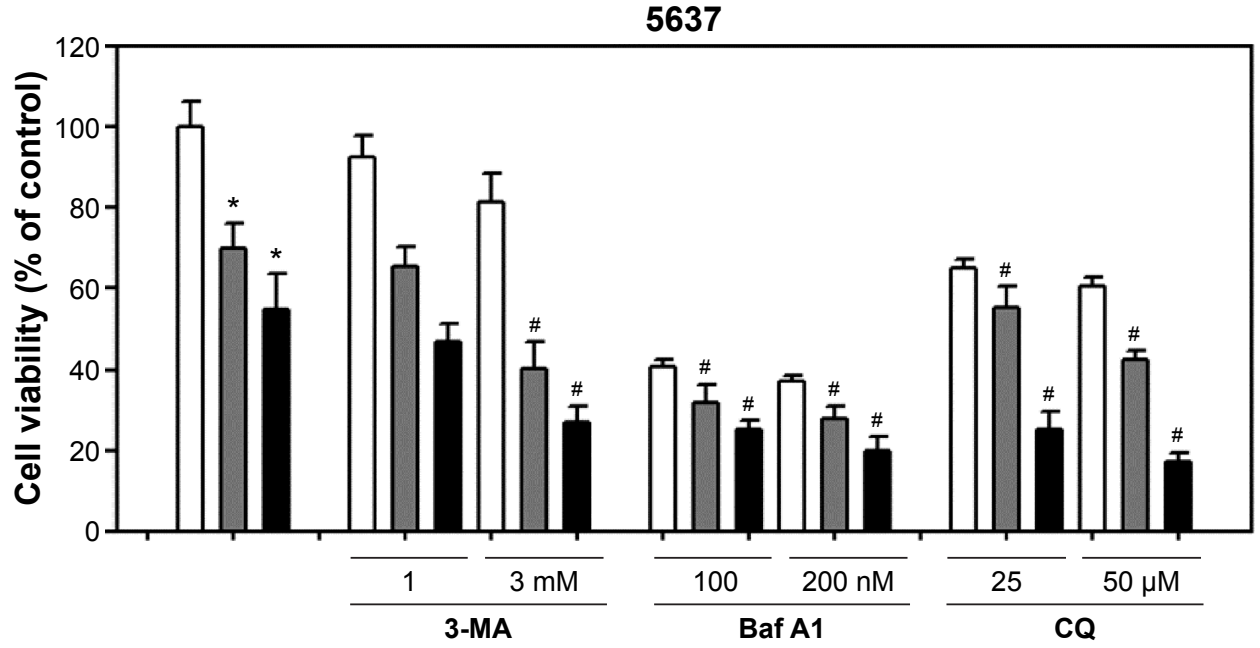

C

T24

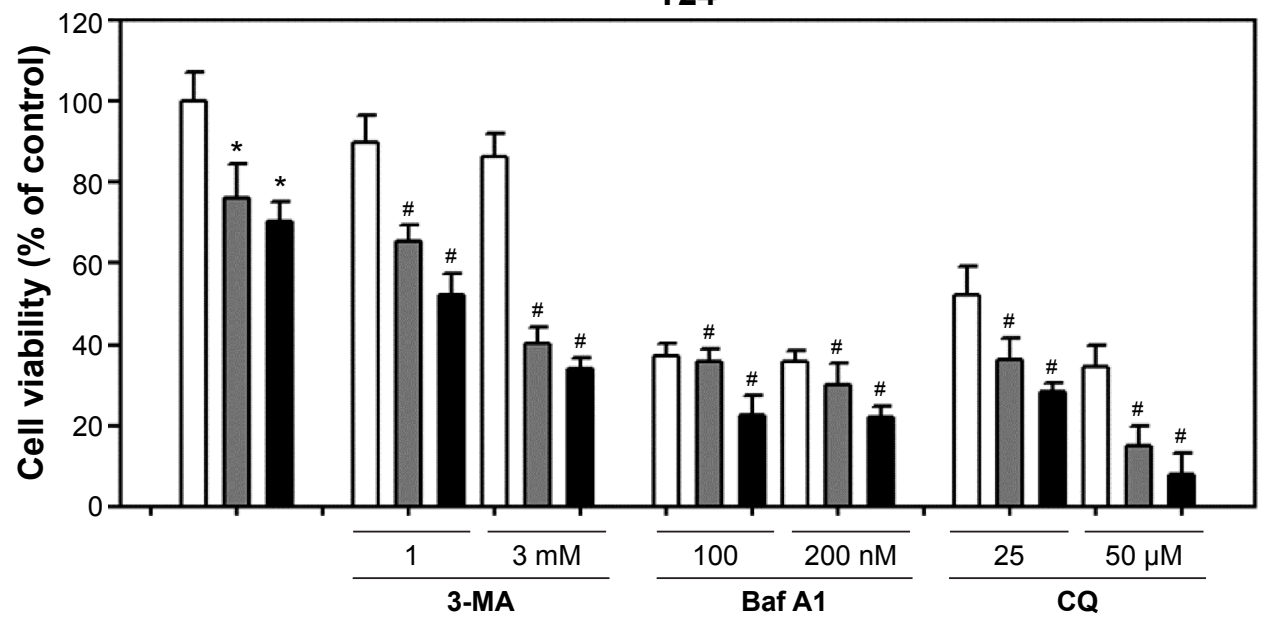

Control

RAD001 $1 \mu \mathrm{M}$

RAD001 $5 \mu \mathrm{M}$

Figure 4 Inhibition of autophagy increases RAD00I-induced cytotoxicity.

Notes: Cell viability of (A) RT4, (B) 5637, and (C) T24 cells treated with I or $5 \mu$ M RAD00I with or without the cotreatment of I or 3 mM $3-M A$, I00 or 200 nM Baf AI, or 25 or $50 \mu \mathrm{M} \mathrm{CQ}$ for 72 hours. Cell viability was detected using WST-8 reagent, and the values are shown as the mean \pm SD of three independent experiments; $* P<0.05$. ${ }^{\#} P<0.05$ when compared to RAD00I treatment group.

Abbreviations: Baf AI, bafilomycin AI; CQ, chloroquine; SD, standard deviation; 3-MA, 3-methyladenine. 


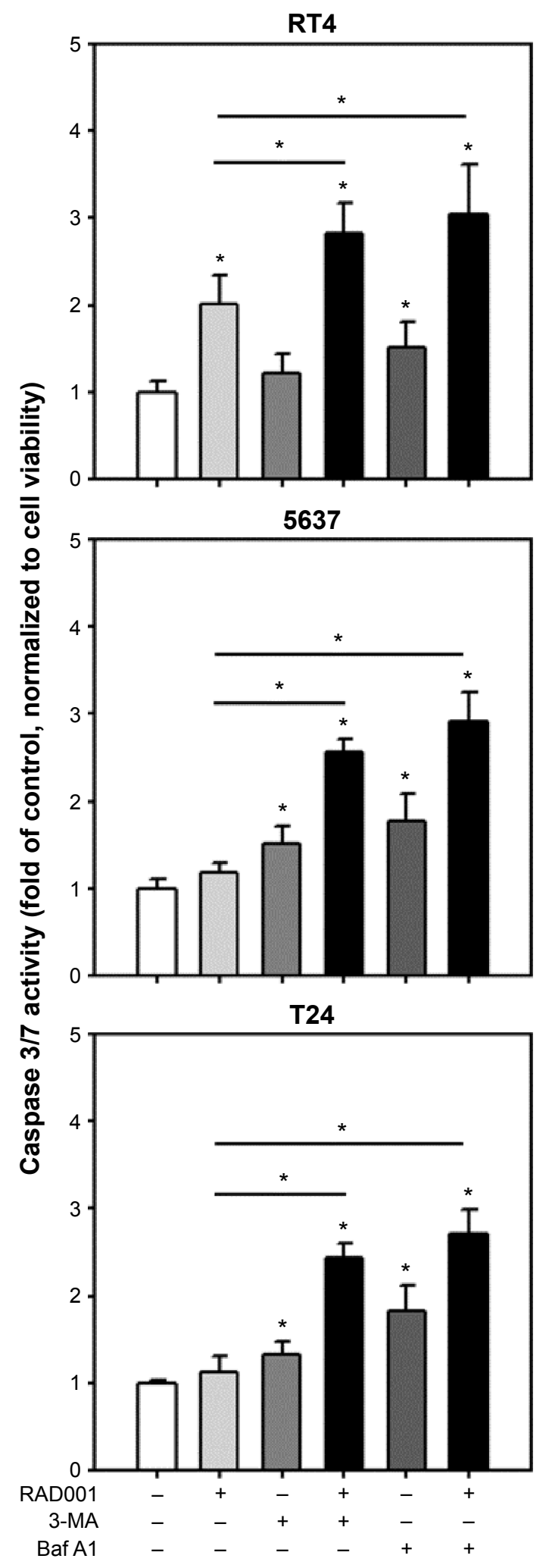

Figure 5 Coordinate inhibition of autophagy and mTOR enhances apoptosis in human bladder cancer cells.

Notes: Caspase $3 / 7$ activity was detected in RT4, 5637, and T24 cells treated with I $\mu$ M RAD00 I with or without the cotreatment of 3 mM 3-MA or $200 \mathrm{nM}$ Baf Al for 24 hours. The data from three independent experiments are shown. The results are presented as the mean $\pm S D ; * P<0.05$.

Abbreviations: $m T O R$, mammalian target of rapamycin; SD, standard deviation; Baf AI, bafilomycin AI; 3-MA, 3-methyladenine. the mTORC2 is suggested to be insensitive to the presence of rapamycin and its derivatives and was shown to regulate the prosurvival kinase AKT by phosphporylation on Ser $473 .{ }^{29}$ Therefore, inhibition of mTORC1 results in inhibition of negative feedback loops and leads to rapamycin resistance in some cell types. ${ }^{30,31}$ Our results showed a decreased expression of mTOR complexes and AKT1 transcripts in RAD001-treated T24 cells, and only the expression of AKT1 was slightly increased upon $5 \mu \mathrm{M}$ RAD001 treatment. Rapamycin and RAD001 are well recognized as specific inhibitors that act on mTORC1, and the inhibition usually accompanies with feedback activation of mTORC2 and AKT prosurvival pathway as already mentioned. Our results from the transcription level may also help to explain why prolonged inhibition of mTORC1 inhibits mTORC2 assembly and AKT activation. ${ }^{32}$ The differences in bladder cancer cells responding to mTOR inhibition may be attributed to other factors, such as variability of mTORC1 and mTORC2 response or activation/inhibition of other pathways upon mTOR inhibition. RAD001 has been shown to activate MAP kinase (MAPK) through S6K/PI3K/Ras signaling, which, in turn, enhances survival of cells. ${ }^{33}$ These complex interplays between $\mathrm{mTOR}$ and other pathways are postulated to account for differences in sensitivity to RAD001.

Autophagy is a fine-tuned catabolic process that is critical in organelle degradation and protein turnover. It is present at low levels in normal cell and upregulated in response to metabolic stresses. It is evident that mTOR (as a sensor of cellular nutritional status, stress, and growth factor signals), particularly mTORC1, plays a role in autophagy signaling pathway. A previous study has demonstrated that autophagy is induced by mTORC1 inhibition, whereas stimulation of mTORC1 inhibits this process. ${ }^{34}$ The mTORC1 was shown to control autophagy by regulation of a protein complex consisting of ULK1, ATG13, and FIP200. Inhibition of mTORC1 resulted in the decreased phosphorylation of ULK1 and ATG13, and therefore induction of autophagy. ${ }^{35}$ Although starvation or stress signaling is not always mediated by mTORC1, and it is possible that other mTORC1-independent pathways regulate ULK complexes, ${ }^{36}$ the current knowledge suggests that mTORC1 and ULK complexes constitute the main axis of the pathways that regulates growth and autophagy. In this study, we observed that RAD001, as an mTORC1 inhibitor, induces autophagy in bladder cancer cells corresponding with variable cytotoxicity. Autophagy was initially considered a process that suppressed malignant transformation. ${ }^{37}$ Activation of the PI3K/ AKT pathway via activating $P I 3 K$ mutations, AKT amplifications, or PTEN loss has been reported to attenuate autophagy 
in many settings largely through mTOR activation. ${ }^{38,39}$ The p53 protein has been shown to have opposing roles in autophagy, which is activated by nutrient deprivation or genotoxic stress leading to activation of autophagy as well as inhibition of mTOR. In contrast, p53 has been demonstrated to suppress autophagy in the basal state..$^{40,41}$

Despite a role in tumor suppression, autophagy has been demonstrated to play a potentiating role in cancer development, promoting survival in cells under a number of stresses. ${ }^{40,42,43}$ In addition, autophagy has been shown to mediate therapeutic resistance in a variety of situations. ${ }^{44}$ For example, our previous study demonstrated that natural chemopreventive compound, benzyl isothiocyanate, induces protective autophagy in human prostate cancer cells. ${ }^{17}$ Furthermore, we reported that high-grade bladder cancer exhibits high basal level of autophagy. ${ }^{45}$ It is possible that inhibition of mTORC1 with RAD001 further enhances autophagy and accounts for the resistance of this drug in highgrade bladder cancer cells. Recent studies have demonstrated that autophagy is induced by restoration of p53 expression or by alkylating chemotherapy and that inhibition of autophagy enhances the antiproliferative activity of chemotherapy. ${ }^{46-48}$ In this study, we showed that combination of RAD001 with autophagy inhibitor leads to significantly increased apoptotic cell death in bladder cancer cells. It is suggested that the mTOR inhibition by RAD001 induces a protective autophagy blocking apoptosis pathway. Our results were consistent with the report that inhibition of mTOR and autophagy coordinately enhances cell death in melanoma. ${ }^{49}$ In addition, a phase I trial of temsirolimus (another mTORC1 inhibitor) and hydroxychloroquine (an autophagy inhibitor that disrupt autophagosomes fusion with lysosomes) demonstrated that the combination of temsirolimus and hydroxychloroquine is safe and tolerable in patients with advanced solid tumors and melanoma. ${ }^{50}$ Therefore, inhibition of autophagy is suggested to enhance RAD001-induced apoptotic cell death in bladder cancer.

\section{Conclusion}

The antiproliferation effect of RAD001 on bladder cancer cells is heterogeneous. Inhibition of autophagy significantly enhances cytotoxicity of RAD001 in bladder cancer cells. Combination of mTOR inhibitor and autophagy inhibitor represents a potential effective approach to manage bladder cancer. Further studies are needed to elucidate the mechanism of $\mathrm{mTOR}$ regulation and its clinical role in the treatment of bladder cancer.

\section{Acknowledgments}

This work was supported by Shin Kong Wu Ho-Su Memorial Hospital, Taipei, Taiwan (SKH-8302-101-DR-06 and
SKH-8302-102-DR-10 to YCL and SKH-8302-101-0202 to JFL).

\section{Disclosure}

The authors report no conflicts of interest in this work.

\section{References}

1. Malats N, Real FX. Epidemiology of bladder cancer. Hematol Oncol Clin North Am. 2015;29:177-189, vii.

2. van der Meijden AP. Optimal treatment for intermediate- and highrisk, nonmuscle-invasive bladder cancer. ScientificWorldJournal. 2006;6:2611-2616.

3. Dovedi SJ, Davies BR. Emerging targeted therapies for bladder cancer: a disease waiting for a drug. Cancer Metastasis Rev. 2009;28:355-367.

4. Hashimoto I, Koizumi K, Tatematsu M, et al. Blocking on the CXCR4/ mTOR signalling pathway induces the anti-metastatic properties and autophagic cell death in peritoneal disseminated gastric cancer cells. Eur J Cancer. 2008;44:1022-1029.

5. Lamouille S, Derynck R. Cell size and invasion in TGF-beta-induced epithelial to mesenchymal transition is regulated by activation of the mTOR pathway. J Cell Biol. 2007;178:437-451.

6. Faried LS, Faried A, Kanuma T, et al. Predictive and prognostic role of activated mammalian target of rapamycin in cervical cancer treated with cisplatin-based neoadjuvant chemotherapy. Oncol Rep. 2006;16: $57-63$.

7. Hansel DE, Platt E, Orloff M, et al. Mammalian target of rapamycin (mTOR) regulates cellular proliferation and tumor growth in urothelial carcinoma. Am J Pathol. 2010;176:3062-3072.

8. Fasolo A, Sessa C. mTOR inhibitors in the treatment of cancer. Expert Opin Investig Drugs. 2008;17:1717-1734.

9. Huang JJ, Li ZM, Huang Y, et al. Schedule-dependent inhibition of T-cell lymphoma cells by cotreatment with the mTOR inhibitor everolimus and anticancer drugs. Invest New Drugs. 2012;30:223-235.

10. Hurvitz SA, Kalous O, Conklin D, et al. In vitro activity of the mTOR inhibitor everolimus, in a large panel of breast cancer cell lines and analysis for predictors of response. Breast Cancer Res Treat. 2015;149: 669-680.

11. Lee SJ, Lee J, Lee J, et al. Phase II trial of capecitabine and everolimus (RAD001) combination in refractory gastric cancer patients. Invest New Drugs. 2013;31:1580-1586.

12. Wedel S, Hudak L, Seibel JM, et al. Inhibitory effects of the HDAC inhibitor valproic acid on prostate cancer growth are enhanced by simultaneous application of the mTOR inhibitor RAD001. Life Sci. 2011; 88:418-424.

13. Motzer RJ, Escudier B, Oudard S, et al. Efficacy of everolimus in advanced renal cell carcinoma: a double-blind, randomised, placebocontrolled phase III trial. Lancet. 2008;372:449-456.

14. Erdemoglu E, Guney M, Take G, Giray SG, Mungan T. RAD001 (Everolimus) can prevent tamoxifen-related endometrial and stromal hyperplasia. Int J Gynecol Cancer. 2009;19:375-379.

15. Liu E, Marincola P, Oberg K. Everolimus in the treatment of patients with advanced pancreatic neuroendocrine tumors: latest findings and interpretations. Therap Adv Gastroenterol. 2013;6:412-419.

16. Vignot S, Faivre S, Aguirre D, Raymond E. mTOR-targeted therapy of cancer with rapamycin derivatives. Ann Oncol. 2005;16:525-537.

17. Lin JF, Tsai TF, Liao PC, et al. Benzyl isothiocyanate induces protective autophagy in human prostate cancer cells via inhibition of mTOR signaling. Carcinogenesis. 2013;34:406-414.

18. Chang CJ, Lin JF, Chang HH, Lee GA, Hung CF. Lutein protects against methotrexate-induced and reactive oxygen species-mediated apoptotic cell injury of IEC-6 cells. PLoS One. 2013;8:e72553.

19. Lin JF, Lin YC, Lin YH, et al. Zoledronic acid induces autophagic cell death in human prostate cancer cells. J Urol. 2011;185:1490-1496.

20. O'Reilly KE, Rojo F, She QB, et al. mTOR inhibition induces upstream receptor tyrosine kinase signaling and activates Akt. Cancer Res. 2006; 66:1500-1508. 
21. Pankiv S, Clausen TH, Lamark T, et al. p62/SQSTM1 binds directly to Atg8/LC3 to facilitate degradation of ubiquitinated protein aggregates by autophagy. J Biol Chem. 2007;282:24131-24145.

22. Huang S, Houghton PJ. Targeting mTOR signaling for cancer therapy. Curr Opin Pharmacol. 2003;3:371-377.

23. Wengrod JC, Gardner LB. Cellular adaptation to nutrient deprivation: crosstalk between the mTORC1 and eIF2alpha signaling pathways and implications for autophagy. Cell Cycle. 2015;14:2571-2577.

24. Tang L, Zirpoli GR, Guru K, et al. Intake of cruciferous vegetables modifies bladder cancer survival. Cancer Epidemiol Biomarkers Prev. 2010;19:1806-1811.

25. Cam M, Bid HK, Xiao L, et al. p53/TAp63 and AKT regulate mammalian target of rapamycin complex 1 (mTORC1) signaling through two independent parallel pathways in the presence of DNA damage. J Biol Chem. 2014;289:4083-4094.

26. Wall M, Poortinga G, Stanley KL, et al. The mTORC1 inhibitor everolimus prevents and treats Emu-Myc lymphoma by restoring oncogene-induced senescence. Cancer Discov. 2013;3:82-95.

27. Zhang WB, Wang Z, Shu F, et al. Activation of AMP-activated protein kinase by temozolomide contributes to apoptosis in glioblastoma cells via p53 activation and mTORC1 inhibition. J Biol Chem. 2010;285: 40461-40471.

28. Akeno N, Miller AL, Ma X, Wikenheiser-Brokamp KA. p53 suppresses carcinoma progression by inhibiting mTOR pathway activation. Oncogene. 2015;34:589-599.

29. Sarbassov DD, Guertin DA, Ali SM, Sabatini DM. Phosphorylation and regulation of Akt/PKB by the rictor-mTOR complex. Science. 2005; 307:1098-1101.

30. Hsu PP, Kang SA, Rameseder J, et al. The mTOR-regulated phosphoproteome reveals a mechanism of mTORC1-mediated inhibition of growth factor signaling. Science. 2011;332:1317-1322.

31. Sabatini DM. mTOR and cancer: insights into a complex relationship. Nat Rev Cancer. 2006;6:729-734

32. Sarbassov DD, Ali SM, Sengupta S, et al. Prolonged rapamycin treatment inhibits mTORC2 assembly and Akt/PKB. Mol Cell. 2006; 22:159-168.

33. Carracedo A, Ma L, Teruya-Feldstein J, et al. Inhibition of mTORC1 leads to MAPK pathway activation through a PI3K-dependent feedback loop in human cancer. J Clin Invest. 2008;118:3065-3074.

34. Codogno P, Meijer AJ. Autophagy and signaling: their role in cell survival and cell death. Cell Death Differ. 2005;12(Suppl 2):1509-1518.

35. Hosokawa N, Hara T, Kaizuka T, et al. Nutrient-dependent mTORC1 association with the ULK1-Atg13-FIP200 complex required for autophagy. Mol Biol Cell. 2009;20:1981-1991.

36. Sarkar S, Ravikumar B, Floto RA, Rubinsztein DC. Rapamycin and mTOR-independent autophagy inducers ameliorate toxicity of polyglutamine-expanded huntingtin and related proteinopathies. Cell Death Differ. 2009;16:46-56.
37. Guertin DA, Sabatini DM. An expanding role for $m T O R$ in cancer. Trends Mol Med. 2005;11:353-361.

38. Lamoureux F, Zoubeidi A. Dual inhibition of autophagy and the AKT pathway in prostate cancer. Autophagy. 2013;9:1119-1120.

39. Cao C, Subhawong T, Albert JM, et al. Inhibition of mammalian target of rapamycin or apoptotic pathway induces autophagy and radiosensitizes PTEN null prostate cancer cells. Cancer Res. 2006;66: 10040-10047.

40. Huo Y, Cai H, Teplova I, et al. Autophagy opposes p53-mediated tumor barrier to facilitate tumorigenesis in a model of PALB2-associated hereditary breast cancer. Cancer Discov. 2013;3:894-907.

41. Lee HY, Chung KJ, Hwang IH, et al. Activation of p53 with ilimaquinone and ethylsmenoquinone, marine sponge metabolites, induces apoptosis and autophagy in colon cancer cells. Mar Drugs. 2015;13 543-557.

42. Toshima T, Shirabe K, Matsumoto Y, et al. Autophagy enhances hepatocellular carcinoma progression by activation of mitochondrial beta-oxidation. J Gastroenterol. 2014;49:907-916.

43. Shi Y, Han JJ, Tennakoon JB, et al. Androgens promote prostate cancer cell growth through induction of autophagy. Mol Endocrinol. 2013; 27:280-295

44. Morselli E, Galluzzi L, Kepp O, et al. Anti- and pro-tumor functions of autophagy. Biochim Biophys Acta. 2009;1793:1524-1532.

45. Lin YC, Lin JF, Wen SI, et al. Inhibition of high basal level of autophagy induces apoptosis in human bladder cancer cells. J Urol. Epub 2015 Oct 28

46. Pal I, Parida S, Prashanth Kumar BN, et al. Blockade of autophagy enhances proapoptotic potential of BI-69A11, a novel Akt inhibitor, in colon carcinoma. Eur J Pharmacol. Epub 2015 Oct 15.

47. Li LQ, Xie WJ, Pan D, Chen H, Zhang L. Inhibition of autophagy by bafilomycin A1 promotes chemosensitivity of gastric cancer cells. Tumour Biol. Epub 2015 Aug 15.

48. Ruan Y, Hu K, Chen H. Autophagy inhibition enhances isorhamnetininduced mitochondriadependent apoptosis in nonsmall cell lung cancer cells. Mol Med Rep. 2015;12(4):5796-5806.

49. Xie X, White EP, Mehnert JM. Coordinate autophagy and mTOR pathway inhibition enhances cell death in melanoma. PLoS One. 2013; 8:e55096.

50. Rangwala R, Chang YC, Hu J, et al. Combined MTOR and autophagy inhibition: phase I trial of hydroxychloroquine and temsirolimus in patients with advanced solid tumors and melanoma. Autophagy. 2014; 10:1391-1402. 


\section{Supplementary material}

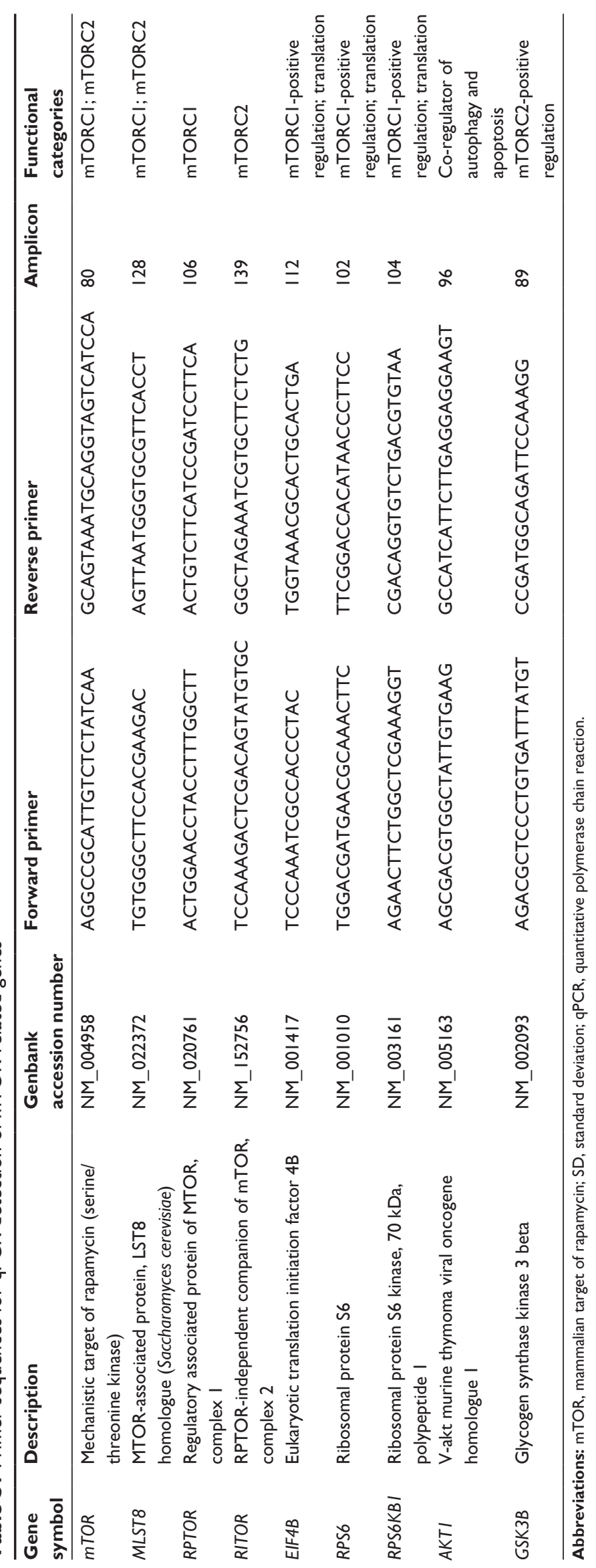




\section{Publish your work in this journal}

Drug Design, Development and Therapy is an international, peerreviewed open-access journal that spans the spectrum of drug design and development through to clinical applications. Clinical outcomes, patient safety, and programs for the development and effective, safe, and sustained use of medicines are a feature of the journal, which has also been accepted for indexing on PubMed Central. The manuscript management system is completely online and includes a very quick and fair peer-review system, which is all easy to use. Visit http://www.dovepress.com/testimonials.php to read real quotes from published authors.

Submit your manuscript here: http://www.dovepress.com/drug-design-development-and-therapy-journal 